\title{
Microstructure and Corrosion Properties of the Plasma-MIG Welded AA5754 Automotive Alloy
}

\author{
A. Abouarkoub ${ }^{1}$, G. E. Thompson ${ }^{2}$, X. Zhou ${ }^{2}$, G. Scamans ${ }^{3}$ \\ ${ }^{1}$ The Libyan Petroleum Institute, Tripoli, Libya \\ ${ }^{2}$ School of Materials, The University of Manchester, Manchester, UK \\ ${ }^{3}$ Innoval Technology Limited, Banbury, UK \\ Email: ${ }^{*}$ abdalhadi aboargoub@yahoo.com
}

Received 25 May 2015; accepted 27 June 2015; published 30 June 2015

Copyright (C) 2015 by authors and Scientific Research Publishing Inc.

This work is licensed under the Creative Commons Attribution International License (CC BY). http://creativecommons.org/licenses/by/4.0/

(c) (7) Open Access

\section{Abstract}

The influence of heating cycles during plasma metal inert gas (MIG) welding on the microstructure and corrosion properties of the AA5754 automotive alloy has been investigated. The high heat input during plasma-MIG welding results in a significant modification in the microstructure of the AA5754 alloy adjacent to the fusion boundaries. As a consequence of partial melting of the Al-Fe-Mn-(Si) intermetallics at the partially melted zone (PMZ) and segregation of the high melting point elements (particularly $\mathrm{Fe}$ and $\mathrm{Mn}$ ) toward the fusion zone, severe galvanic corrosion attacks can be enhanced along the PMZ of the AA5754 weld during exposure to aqueous corrosion environments.

\section{Keywords}

Plasma-MIG Welding, AA5754, Microstructure, Corrosion, Partially Melted Zone

\section{Introduction}

In the automotive industry, welding is one of the most critical elements of the body assembly process, which determines the structural integrity and quality of the vehicles being manufactured. The microstructure of the automotive aluminium wrought alloys can be significantly modified by the high heat input employed during fusion welding techniques, such as the plasma MIG welding [1]-[3]. However, different from the heat treatable aluminium-alloys of the $2 \mathrm{xxx}, 6 \mathrm{xxx}$ and $7 \mathrm{xxx}$ series, the change in the mechanical properties of aluminiummagnesium based 5xxx alloys is not significant except for the cold worked alloys, where the mechanical

"Corresponding author.

How to cite this paper: Abouarkoub, A., Thompson, G.E., Zhou, X. and Scamans, G. (2015) Microstructure and Corrosion Properties of the Plasma-MIG Welded AA5754 Automotive Alloy. Journal of Minerals and Materials Characterization and Engineering, 3, 318-325. http://dx.doi.org/10.4236/jmmce.2015.34034 
strength can be slightly reduced. There are, however, a number of corrosion issues associated with the formation of various weld zones through fusion welding of the automotive aluminium-magnesium alloys that may deteriorate the performance of the car body connections during service. One of the most critical defects induced by fusion welding, which may contribute to the loss of mechanical and corrosion properties, is the inhomogeneity near the fusion boundary. This phenomenon is often encountered in dissimilar welding of aluminium alloys, where filler metal of different composition from the base metal is used, or when two base metals of different composition are fusion welded together. In such a case, the chemical composition, microstructure and mechanical properties of the partially melted zone near the fusion boundary may differ significantly from the weld and the parent metals [4] [5]. The modification in the chemical composition of the partially melted zone relative to the base metal can be related to solute segregation during the initial transient of solidification, which has been frequently observed in various welds, including steel, stainless steels and aluminium alloys [6] [7]. The composition gradients through the base metal, partially melted zones and the fusion zone of the commercial $5 \mathrm{xxx}$ alloys, such as AA5754, are often caused by the rejection of high melting point impurities, for example iron and manganese, from the newly forming solid weld metal into the melt during the initial stage of solidification. This phenomenon, in turn, is expected to modify the corrosion behaviours and, thus, the overall performance of these alloys during service.

\section{Experimental}

The weld samples in the present work were provided by Jaguar Land Rover in the form of a plasma butt weld made of 2 mm AA5754-O panels using AA4043A filler wire "as shown in Figure 1(a)".

For metallographic examination, the investigated welds were first cross-sectioned, polished using conventional grinding and polishing methods followed by etching for $15-20 \mathrm{~s}$ at ambient temperature in Keller's reagent solution ( $85 \mathrm{ml} \mathrm{H}_{2} \mathrm{O}, 10 \mathrm{ml} \mathrm{H}_{2} \mathrm{SO}_{4}$ and, $5 \mathrm{ml} \mathrm{HF}$ ) or $15-20 \mathrm{~s}$ of electro-etching at $20 \mathrm{~V}$ in Barker's reagent (4 $\mathrm{ml}$ hydrofluoric acid and $200 \mathrm{ml} \mathrm{H}_{2} \mathrm{O}$ ).

The influence of the welding cycle on the mechanical properties of the plasma welded alloys was investigated by conducting Vickers microhardness testing across the polished weld sections (parallel to the weld interface) using a Buehler microhardness tester unit. The microhardness measurements were performed using a $0.25 \mathrm{~kg}$ load with a $15 \mathrm{~s}$ dwell time.

For corrosion investigation, several $10 \times 2 \mathrm{~cm}$ samples of the AA5754 plasma weld were cut. Further, in order to eliminate any possible interference of sample geometry with the immersion test results, and to simulate the final surface preparation (prior to painting) in the automotive industry, parts of cut AA5754 plasma welds were mechanically polished until the upper surface was completely flattened (the weld cup was removed), as schematically "shown in Figure 1(b)".

Prior to the immersion test, the transverse sections of the cut samples were mechanically polished to $5 \mu \mathrm{m}$ finish, degreased, washed with water, rinsed with acetone, and then masked with a suitable chemically resistant lacquer (lacomite solution), which was allowed to dry at room temperature for $48 \mathrm{~h}$.

The localized corrosion behaviour of the AA5754 plasma welds was then assessed using a standard accelerated corrosion test in accordance to the G66 ASTM standard by a continuous immersion in in a solution con-

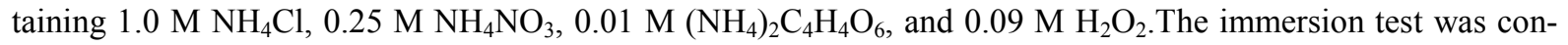
ducted at $65^{\circ} \mathrm{C} \pm 1{ }^{\circ} \mathrm{C}$ in a one litre glass vessel and the solution temperature was precisely controlled during experiments by using a thermostatically controlled water bath.

After the immersion test, samples were directly cleaned with a mixture of methanol and deionized water in an ultrasonic bath for $2 \mathrm{~min}$. Samples for study by scanning electron microscopy, to reveal the morphology and severity of corrosion attack, were cross-sectioned and mechanically polished to a $1 \mu \mathrm{m}$ surface finish.

\section{Results and Discussion}

\subsection{Microstructure Characterization of the AA5754 Plasma-MIG Weld}

The optical micrographs of Figure 2(a) and Figure 3(b) show the cross-sectional features of the AA5754 plasma-MIG butt weld after, respectively, electro-etching at $20 \mathrm{~V}$ in Barker's reagent solution (4 ml hydrofluoric acid and $200 \mathrm{ml} \mathrm{H}_{2} \mathrm{O}$ ) and chemical etching in Keller's reagent $\left(85 \mathrm{ml} \mathrm{H}_{2} \mathrm{O}, 10 \mathrm{ml} \mathrm{H}_{2} \mathrm{SO}_{4}\right.$ and, $5 \mathrm{ml} \mathrm{HF}$ ). In general, the following three different weld zones can be readily distinguished in the etched weld section: 1) the fusion zone "Figure 2 (b)"; 2) the partially melted zone "Figure 2(c)"; 3) the parent metal "Figure 2(d)". 


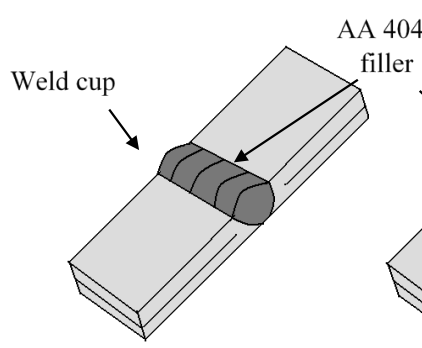

(a)

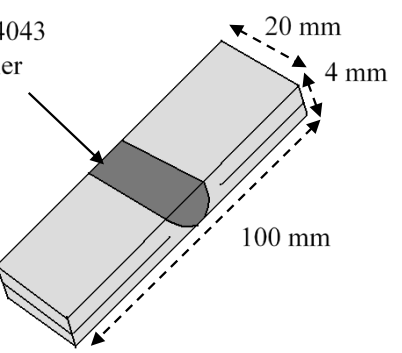

(b)

Figure 1. Schematic diagram of immersion test samples of the AA5754 plasma butt weld: (a) as received sample; (b) mechanically polished sample.

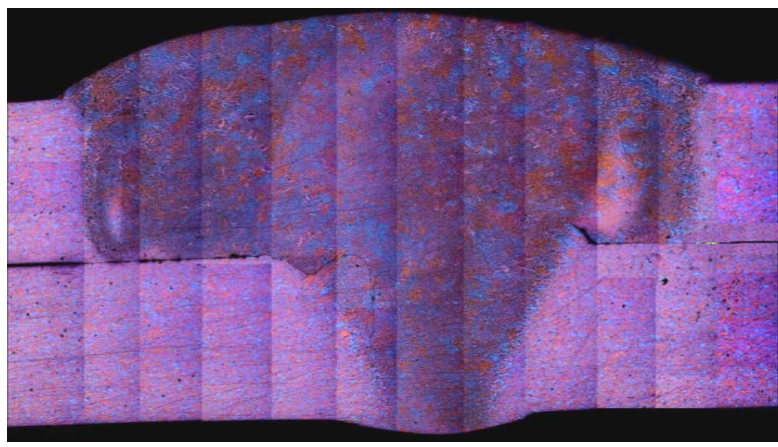

(a)

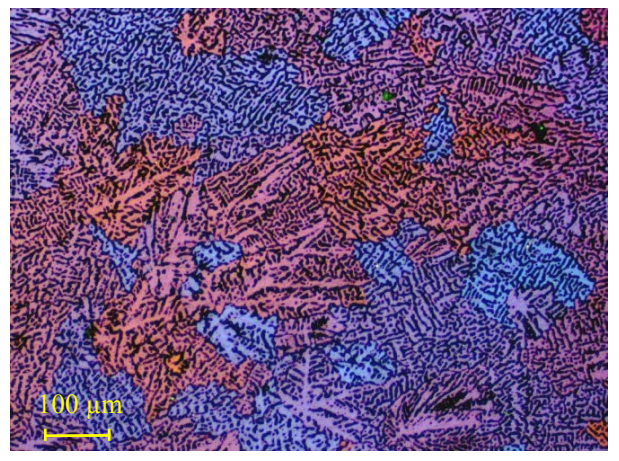

(b)

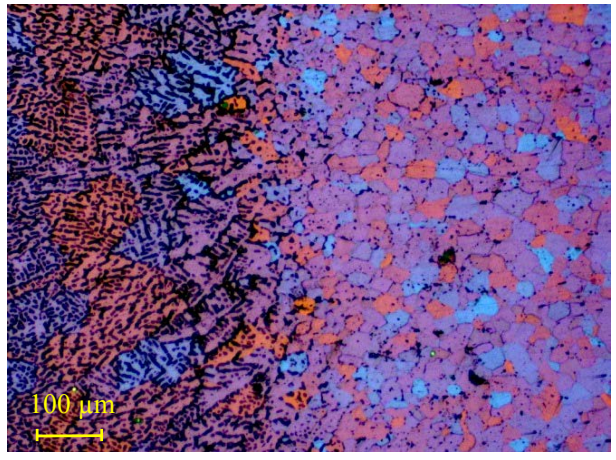

(c)

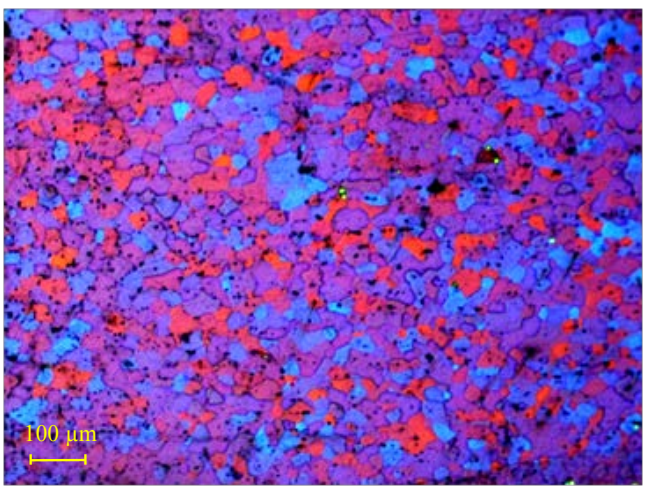

(d)

Figure 2. Optical cross-sectional micrographs of the plasma-MIG AA5754 weld after electroetchingat $20 \mathrm{~V}$ in Barker's reagent (4 $\mathrm{ml}$ hydrofluoric acid and $200 \mathrm{ml} \mathrm{H}_{2} \mathrm{O}$ ): (a) an over-view; (b) fusion zone; (c) the partially melted zone; (d) the parent metal. 


\subsubsection{The Fusion Zone}

The fusion zone of the plasma-MIG weld is the region where the parent metal is completely melted and mixed with the filler alloy material. Upon cooling, solidification of the fusion zone takes place first at the partially melted or solid grains along the fusion boundary (i.e. at the solid-liquid interface). The growth of the solidified material then proceeds toward the fusion centre (i.e. epitaxial growth opposite of the thermal gradient across the weld regions [2]), resulting in the formation of a cellular dendritic Al-Mg-Si structure "Figure 3(b) and Figure 3 (c)" of around $150 \mu \mathrm{m}$ width along the fusion zone boundaries. As solidification continues toward the fusion zone, heterogeneous nucleation of new grains [4] leads to the formation of a dendritic structure of equiaxed grains at the weld centre "Figure 3(d)".

\subsubsection{The Partially Melted Zone}

Next to the fusion boundary of the AA5754 plasma-MIG weld, there is a partially melted structure of about 300 $\mu \mathrm{m}$ width "Figures 3(a)-(c)". The partial melting within this zone resulted in the formation of eutectic-phases at the grain boundaries of the parent material. The peak temperature in this region during welding is expected to exceed the equilibrium solidus or the eutectic temperature for the Al-Mg-Si eutectic constituents. However, the temperature across this region was not sufficient to fully dissolve phases containing high melting point elements (particularly those containing $\mathrm{Fe}$ and $\mathrm{Mn}$ ). As a consequence, partial dissolution of Fe-rich constituents in this zone was detected "Figure 3(e)". Such phenomena may lead to the migration of undissolved solutes (solid state atomic diffusion) toward the fusion zones. In turn, this may cause severe variation in the chemical composition between the PMZ and the adjacent areas.

\subsubsection{The Parent Metal}

The parent metal adjacent to the partially melted zone of the AA5754 plasma-MIG weld showed no evidence of over-heating (e.g. excessive grain growth, dissolution or coarsening of second phase particles "Figure 2(d)"and/ or softening "Figure 4".

\subsection{Corrosion Assessment of the AA5754 Plasma-MIG Weld}

The optical micrographs in Figures 5(a)-(d) show the appearance of the as-received "Figure 5(a) and Figure 5(c)" and mechanically polished "Figure 5(b) and Figure 5(d)" AA5754 plasma weld after room temperature immersion in an acidified/chloride solution for $24 \mathrm{~h}$. In general two types of localized attack were detected in the plasma welded samples, namely severe localized corrosion following the upper edges, and large deep pits randomly distributed over the parent metal.

Based on its severity and shape, the first type of localized corrosion attack was identified as a "knife-like attack". Detailed investigation using scanning electron microscopy revealed that the knife-like attack was preferentially initiated and propagated more than $300 \mu \mathrm{m}$ within specific areas just outside and parallel to the fusion zone "Figure 6(a) and Figure 6(b)". The preferential propagation of severe localized attack parallel to the AA5754 alloy weld can be related to the presence of a narrow partially melted zone within which high melting point elements, particularly iron and manganese, were ejected into the melt during the initial stage of solidification. This was evident from the microstructural analysis of the AA5754 plasma weld prior to the immersion test. Partial dissolution of Fe-Mn rich constituents in this region followed by segregation of iron and manganese towards the fusion zone cause severe composition gradients and, thus, potential gradients through the weld zones "Figures 7(a)-(d)". These introduce a galvanic corrosion susceptibility of the plasma welded 5754 alloy in corrosive environments. Form the corrosion view point, the partially melted zone, which is more depleted in iron and manganese compared with the fusion and heat affected zones, is more prone to galvanic attack because its open circuit potential must be shifted to more negative values. Further, the saturation of the fusion zone matrix with elemental silicon (from the $4 \mathrm{xxx}$ filler) normally shifts the open circuit potential to more anodic values. As a consequence, this area can be sacrificially corroded in the presence of a conducting, corrosive electrolyte, where the galvanic circuit elements are all established. Furthermore, in the immersion test, the exposed surface area of the PMZ is very small compared with the more noble adjacent areas; this means that the cathodic galvanic currents may be extremely high, which certainly enhance the severity of galvanic corrosion.

The second type of attack was detected only under areas covered with loose, white scale, which suggests that the scale build up over certain areas during the immersion test somehow initiates such localized corrosion. Based 

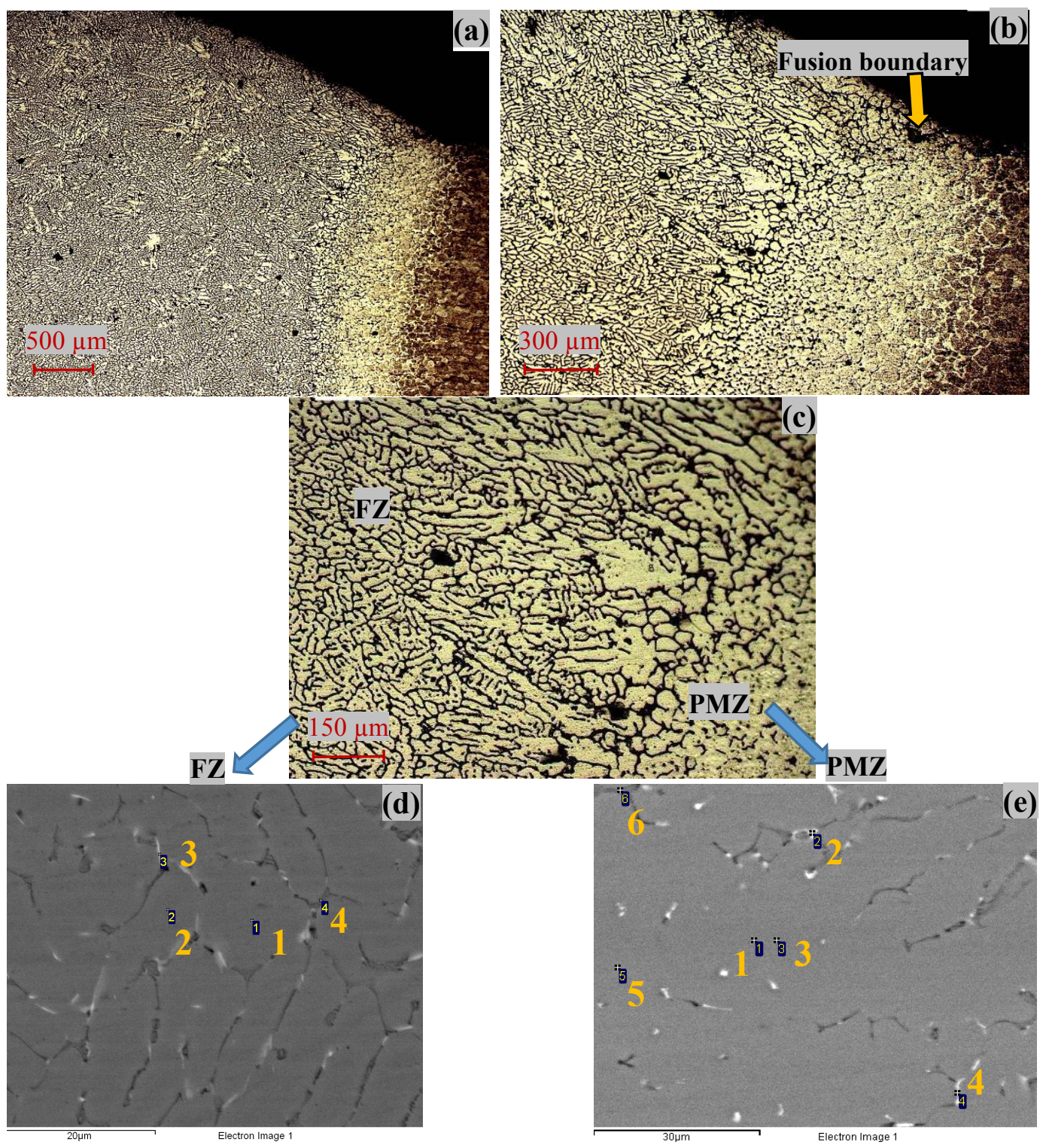

\begin{tabular}{ccccccc}
\hline & O & Mg & Al & Si & Mn & Fe \\
\hline 1 & & 1.73 & 98.27 & & & \\
2 & 3.55 & 1.64 & 94.46 & 0.02 & 0.21 & 0.12 \\
3 & & 2.74 & 96.79 & 0.74 & & \\
4 & 4.60 & 6.22 & 85.38 & 3.81 & & \\
\hline
\end{tabular}

\begin{tabular}{ccccccc}
\hline & $\mathbf{O}$ & $\mathbf{M g}$ & $\mathbf{A l}$ & $\mathbf{S i}$ & $\mathbf{M n}$ & $\mathbf{F e}$ \\
\hline 1 & & 2.15 & 97.85 & & & \\
2 & 3.90 & 5.15 & 87.11 & 0.91 & 0.61 & 2.32 \\
3 & & 2.95 & 97.05 & & & \\
4 & & 4.01 & 95.13 & & 0.44 & 0.42 \\
5 & & 2.51 & 97.19 & & 0.29 & \\
6 & 9.51 & 8.76 & 78.89 & 2.73 & 0.12 & \\
\hline
\end{tabular}

Figure 3. ((a)-(c)) Optical micrographs of increased magnifications of the partially melted zone in the plasma-MIG AA5754 weld after etching in Keller's reagent $\left(85 \mathrm{ml} \mathrm{H}_{2} \mathrm{O}, 10 \mathrm{ml} \mathrm{H}_{2} \mathrm{SO}_{4}\right.$ and, $\left.5 \mathrm{ml} \mathrm{HF}\right)$ ); and ((d), (e)) back scattered electron micrographs and corresponding EDX spot analysis of the eutectic phases at the fusion and partially melted zones, respectively.

on these findings, the second type of corrosion was identified as "under deposit pitting attack". The severity of under deposit attack is better represented in Figure 6(c) and Figure 6(d), where a pit extended horizontally and vertically, and reached a final depth of approximately $1 \mathrm{~mm}$. In this investigation, however, no evidence of any microstructural or chemical variations across the tested samples can be currently related to the initiation or 


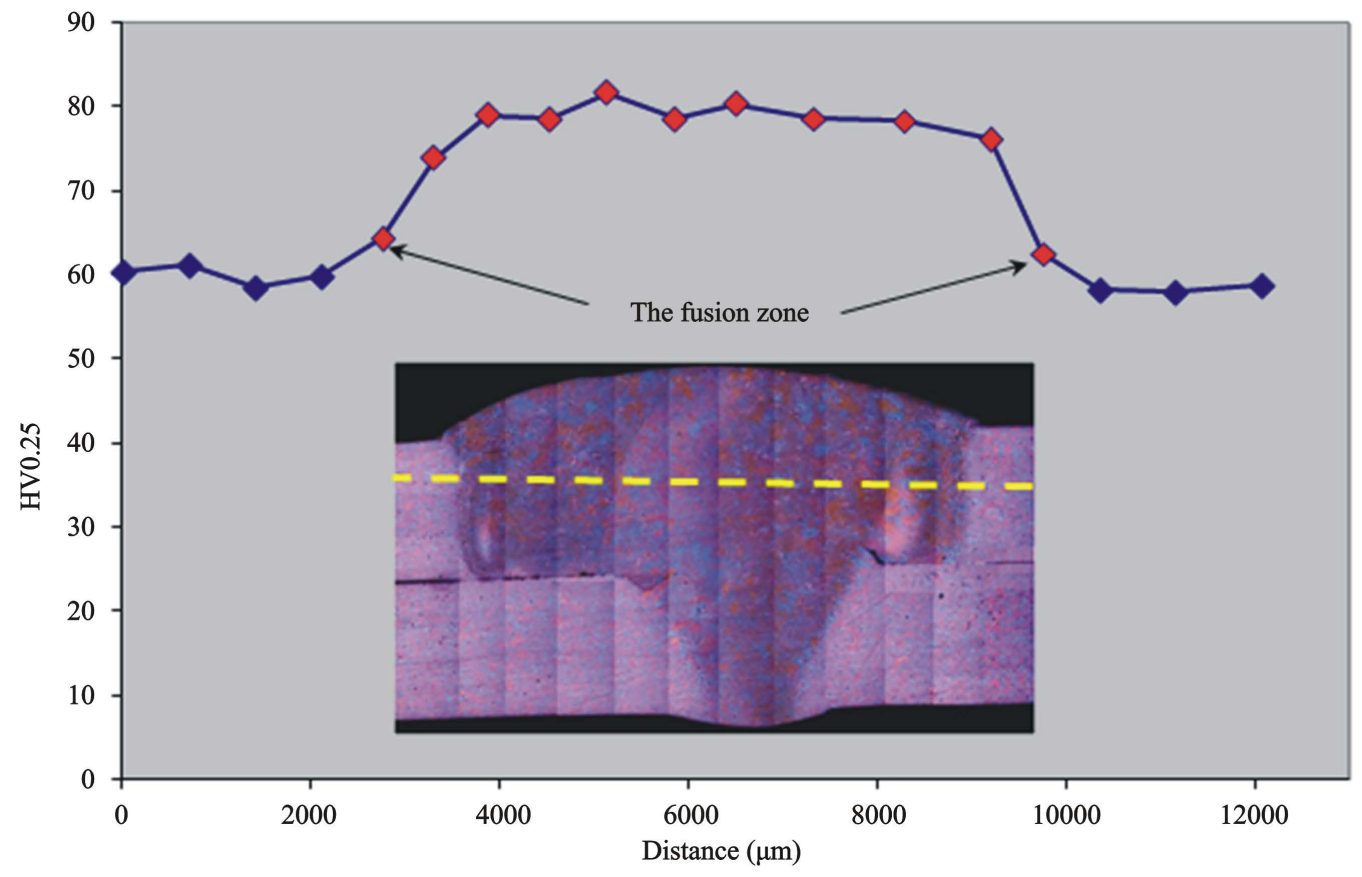

Figure 4. Vickers microhardness profile along the cross-section of the AA5754 plasma-MIG weld.

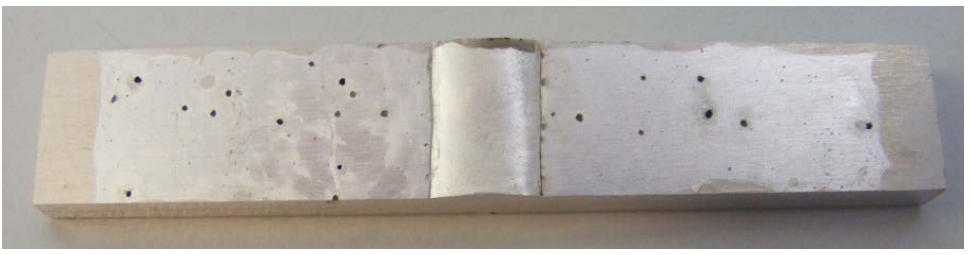

(a)

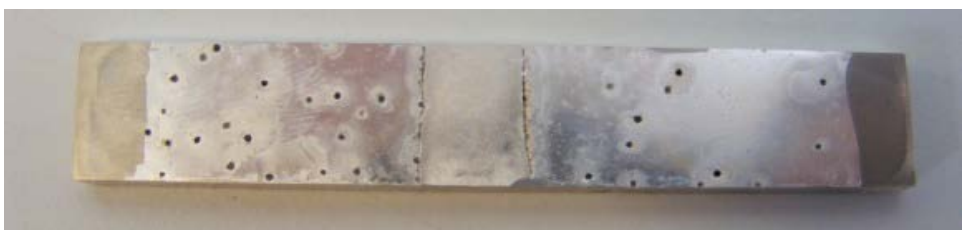

(b)

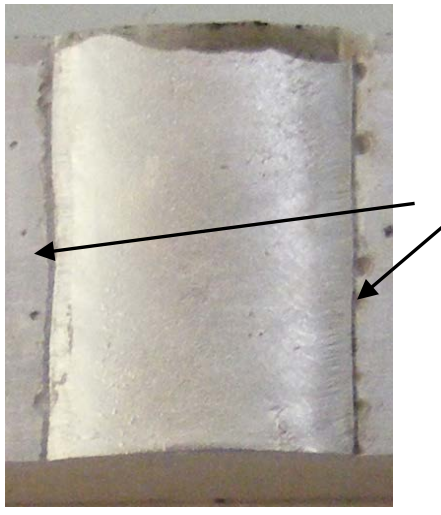

(c)

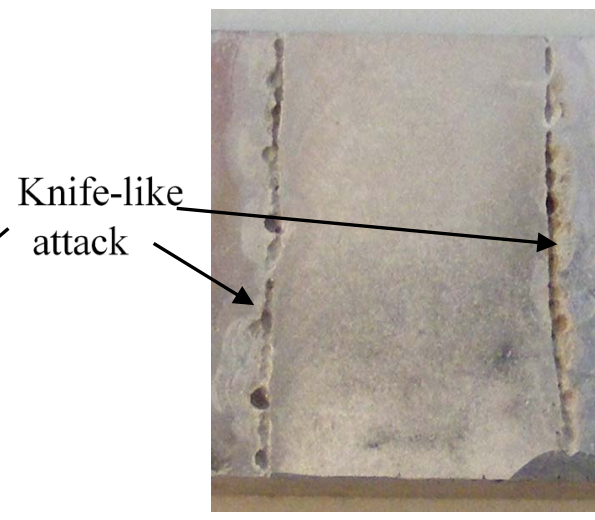

(d)

Figure 5. General view of the AA5754 plasma-MIG butt weld after 24 hours of room temperature immersion in an acidified/chloride solution: ((a), (c)) as-received; ((b), (d)) mechanically polished. 


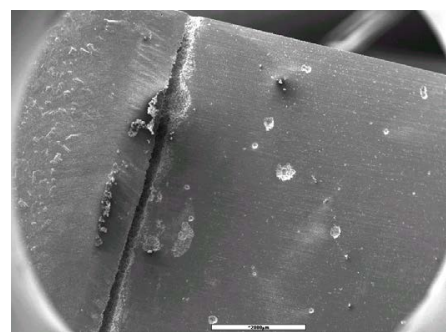

(a)

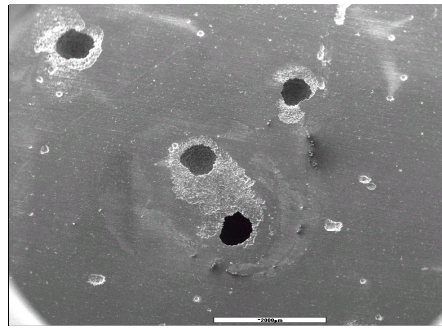

(c)

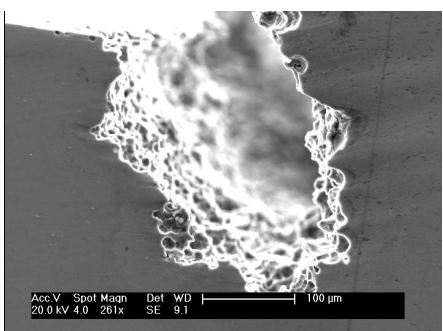

(b)

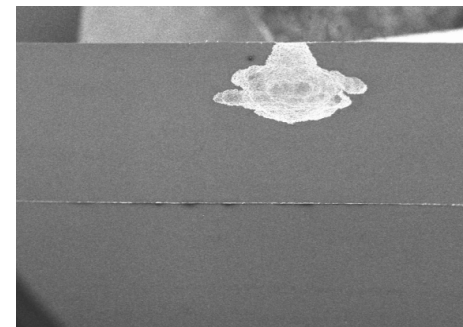

(d)

Figure 6. Top and cross-sectional views of : ((a), (b)) the knife-like attack at the weld edges; and ((c), (d)) severe pitting attack at the parent metal of the AA5754 at plasma butt weld after 24 hours of room temperature immersion in an acidified/chloride solution.

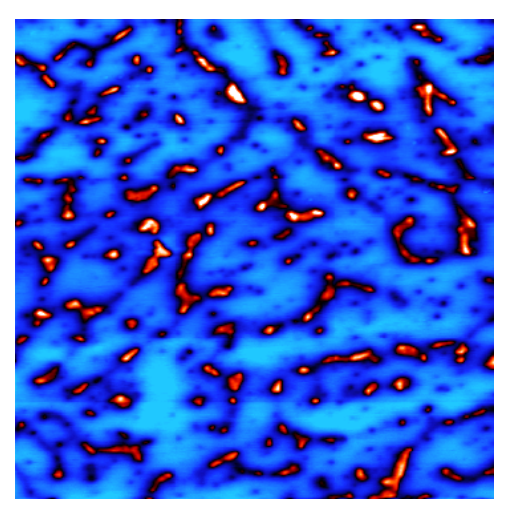

(a)

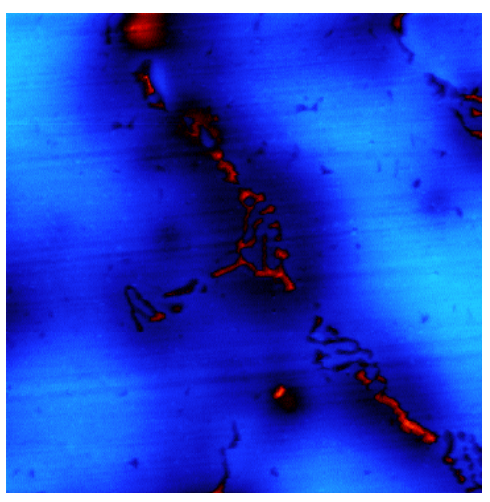

(c)

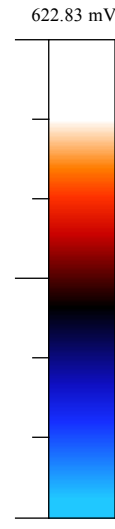

$-170.86 \mathrm{mV}$

$182.86 \mathrm{mV}$

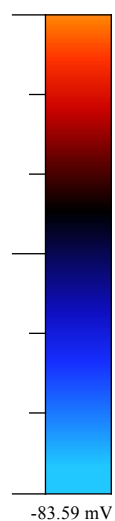

$-83.59 \mathrm{mV}$

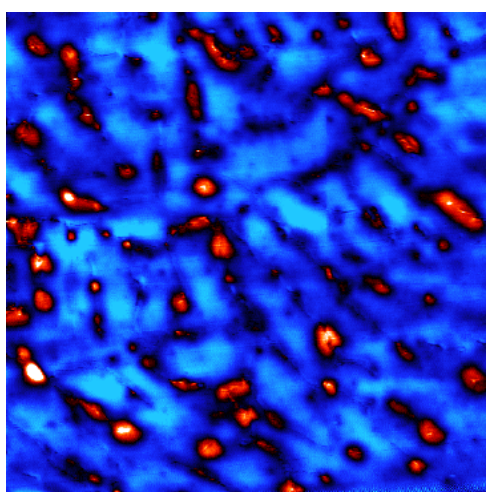

(b)

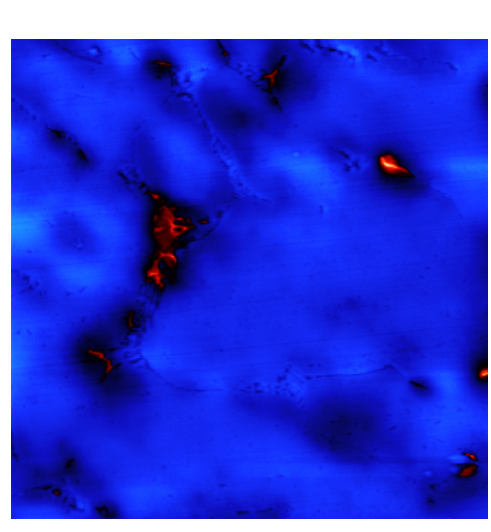

(d)

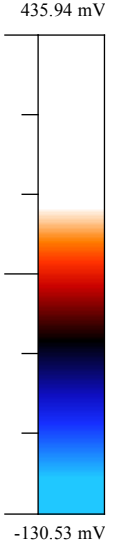

$446.60 \mathrm{mV}$

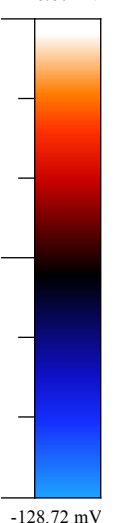

$-128.72 \mathrm{mV}$

Figure 7. AFM mapping across the various weld zones of a polished AA5754 plasma-MIG weld: (a) the fusion zone; (b) the fusion/partially melted zone boundary; (c) the partially melted zone; and (d) partially melted zone/parent metal boundary. 
propagation of the under deposit attack at the parent metal. Most probably, the scale deposition and, thus, the initiation of this attack are related to the local alkalinity around cathodic intermetallics at the samples surface.

\section{Conclusion}

Plasma-MIG welding of the AA5754 alloy using silicon-rich filler wire (AA4043A) results in the formation of a partially melted zone adjacent to the fusion boundaries. Partial melting of the Al-Fe-Mn-(Si) intermetallics at the partially melted zone, and segregation of the high melting point elements (particularly Fe and Mn) towards the fusion zone increase the open circuit potential difference between the weld and its adjacent partially melted zones. Consequently, severe galvanic corrosion attacks take place preferentially along the PMZ at each side of AA5754 weld during exposure to aqueous corrosion environments.

\section{Acknowledgements}

The authors thank EPSRC for support of the LATEST 2 Programme Grant.

\section{References}

[1] Totten, G.E. and MacKenzie, D.S., Eds. (2003) Handbook of Aluminium: Vol. I: Physical Metallurgy and Processes. CRC Press, New York, 481-532.

[2] Kou, S. (2003) Welding Metallurgy. 2nd Edition, Wiley Interscience, New Jersey.

[3] Mathers, G. (2002) The Welding of Aluminium and Its Alloys. Woodhead Publishing Ltd., Cambridge.

[4] Yang, Y. and Kou, S. (2007) Mechanisms of Microsegregation Formation near Fusion Boundary in Welds Made with Dissimilar Filler Metals. Materials Science \& Technology 2007 Conference and Exhibition, Detroit, September 16-20 2007, 329-340.

[5] Kou, S. and Yang, Y. (2007) Fusion-Boundary Macrosegregation in Dissimilar-Filler Welds. Welding Journal, 86, 303-s-312-s.

[6] Baeslack III, W., Lippold, J. and Savage, W. (1979) Unmixed Zone Formation in Austenitic Stainless Steel Weldments. Welding Journal, 58, 168s-176s.

[7] Savage, W., Nippes, E. and Szekeres, E. (1976) A Study of Fusion Boundary Phenomena in a Low Alloy Steel. Welding Journal, 55, 260s-268s. 